\title{
Nonlinear Optical Phenomena in a Silicon-Smectic A Liquid Crystal (SALC) Waveguide
}

\author{
Boris I. Lembrikov *, David Ianetz and Yosef Ben-Ezra \\ Faculty of Electrical Engineering, Holon Institute of Technology, P.O. Box 305, 52 Golomb str., Holon 58102, Israel \\ * Correspondence: borisle@hit.ac.il; Tel.: +972-3-502-6684
}

Received: 13 May 2019; Accepted: 22 June 2019; Published: 28 June 2019

\begin{abstract}
Liquid crystals (LCs) are organic materials characterized by the intermediate properties between those of an isotropic liquid and a crystal with a long range order. The LCs possess strong anisotropy of their optical and electro-optical properties. In particular, LCs possess strong optical nonlinearity. LCs are compatible with silicon-based technologies. Due to these unique properties, LCs are promising candidates for the development of novel integrated devices for telecommunications and sensing. Nematic liquid crystals (NLCs) are mostly used and studied. Smectic A liquid crystals (SALCs) have a higher degree of long range order forming a layered structure. As a result, they have lower scattering losses, specific mechanisms of optical nonlinearity related to the smectic layer displacement without the mass density change, and they can be used in nonlinear optical applications. We theoretically studied the nonlinear optical phenomena in a silicon-SALC waveguide. We have shown theoretically that the stimulated light scattering (SLS) and cross-phase modulation (XPM) caused by SALC nonlinearity can occur in the silicon-SALC waveguide. We evaluated the smectic layer displacement, the SALC hydrodynamic velocity, and the slowly varying amplitudes (SVAs) of the interfering optical waves.
\end{abstract}

Keywords: silicon photonics; optical waveguide; smectic A liquid crystal (SALC); stimulated light scattering (SLS)

\section{Introduction}

Liquid crystals (LCs) are promising candidates for applications in novel integrated devices for telecommunications, sensing, and lab-on-chip bioscience [1]. These applications are based on the unique optical properties of LC. The orientational energy of LC molecules is comparatively small, and for this reason they are characterized by an easy susceptibility to external field perturbation [2]. As a result, the LC effective refractive index can be controlled by an external electric field which may be used for optical transmission, reflection, switching, and modulation applications [2]. LCs are highly nonlinear optical materials because their properties such as temperature, molecular orientation, density, and electronic structure can be easily perturbed by an applied optical field [2].

The liquid crystal on silicon technology (LCOS) is widely used in telecommunications [3,4]. The basic element of the LCOS technology is the LCOS cell consisting of the LCOS backplane, LC layer and cover glass $[3,4]$. The LCOS cell can simultaneously perform the electrical and optical functions [3,4]. The photonic applications of the LCOS devices include the spatial light modulation (SLM), the holographic beam steering, optical wavelength selective switching, and the optical power control [4]. The LCOS SLM technology is a promising candidate for the so-called structured light where the optical field amplitude, phase, and polarization can be controlled spatially while the time and frequency spectrum can be controlled temporally [3]. Nonlinear silicon photonics can be used in on-chip optical signal processing and computation due to its low cost and compatibility with CMOS technology [5]. The development of nonlinear silicon photonics is limited by the absence of the 
second-order nonlinear susceptibility $\chi^{(2)}$ due to centrosymmetric structure of $\mathrm{Si}$, comparatively low third-order nonlinear susceptibility $\chi^{(3)}$, the two-photon absorption (TPA) and free carrier absorption (FCA) [5]. To mitigate these disadvantages new materials with better nonlinear properties may be integrated with silicon. In such a case, the new materials may improve the nonlinearity of an optical device while silicon can confine the optical modes to nanoscale [5]. The organic nonlinear materials with a large $\chi^{(3)}$ can be used for the creation of a silicon-organic hybrid waveguide [5]. In particular, liquid crystals (LCs) may be used as a waveguide core where the modulation and switching of photonic signals is possible by using electro-optic or nonlinear optic effects [1,6-13].

We briefly discuss the basic properties of LCs. LCs are characterized by the properties intermediate between solid crystalline and liquid phases $[2,14]$. LCs flow like liquids, but possess a partial long range order and anisotropy of their physical parameters such as dielectric constants, elastic constants, viscosities, nonlinear susceptibilities [2]. Various phases in which such materials can exist are called mesophases [2]. There are three types of LCs: thermotropic LCs, polymeric LCs, and lyotropic LCs $[2,14]$.

(1) Lyotropic LCs can be obtained in a solution with an appropriate concentration of a material.

(2) Polymeric LCs are the polymers consisting of the monomer LC molecules.

(3) Thermotropic LCs self-assemble in various ordered arrangement of their crystalline axis depending on the temperature.

Thermotropic LCs are most widely used and studied because of their extraordinary linear, electro-optical, and nonlinear optical properties and the possibility to control the transitions between different mesophases by varying the operating temperature [1,2]. The thermotropic LCs consist of elongated molecules with the direction of their axes determined by the unit vector $\vec{n}$ called director [2,14]. The long range ordering of LC mesophase is characterized by the director spatial distribution [2,14]. There exist three main types of thermotropic LCs: nematic LC (NLC), cholesteric LC (CLC), and smectic LC (SLC) [2,14]. NLC molecules are centrosymmetric in such a way that $\vec{n}$ and $-\vec{n}$ are equivalent, the molecules are positionally random, but they are mostly aligned in the direction defined by the director $\vec{n}[2,14]$. CLC consists of chiral molecules, or they may be obtained by adding of chiral molecules to NLC [2]. As a result, they exhibit a helical structure where the direction $\vec{n}$ of the molecular orientation rotates in space around the helical axis with a period of about $300 \mathrm{~nm}$ [2]. The phase transition between the nematic and smectic A phases had been investigated both theoretically and experimentally in a large number of publications (see, for example, [2,15-22]). It is essentially the second kind phase transition [18]. The phase transition temperature $T_{S m A-N}$ may be different for different LC materials. For example, for $8 \mathrm{CB} T_{S m A-N} \approx 307 \mathrm{~K}$, for $9 \mathrm{CB} T_{S m A-N} \approx 321 \mathrm{~K}$ [22].

SLC are characterized by the positional long range order in the direction of the elongated molecular axis and possess a layered structure with a layer thickness of about $2 \mathrm{~nm}$ approximately equal to the length of a SLC molecule [2,14]. Inside the layers, molecules are not ordered and represent a two-dimensional liquid $[2,14]$. There are different SLC phases $[2,14]$.

(1) Smectic A LC (SALC) where the long axes of the molecules are perpendicular to the layer plane.

(2) Smectic B LC where the hexagonal in-layer ordering of the molecules perpendicular to the layer plane exists.

(3) Smectic C LC where the molecules are tilted with respect to the layers.

(4) Smectic $C^{*}$ LC consisting of the chiral molecules and possessing the spontaneous polarization.

(5) So-called exotic smectic phases.

The nonlinear optical phenomena such as degenerate and nondegenerate wave mixing, optical bistabilities and instabilities, self-focusing and self-guiding, phase conjugation, stimulated light scattering (SLS), optical limiting, interface switching, beam combining, and self-starting laser oscillations have been observed in liquid crystalline materials [14,23]. NLCs are mainly studied and 
used in linear and nonlinear optical applications [2,4,23]. For instance, in NLC the optically induced director axis reorientation results in the so-called giant optical nonlinearity (GON) with the nonlinear refractive index coefficient $n_{2}^{N L} \sim 10^{-4}-10^{-3} \mathrm{~cm}^{2} / \mathrm{W}$ [14]. However, NLCs are characterized by large losses and relatively slow responses limiting their integrated electro-optical applications [2]. The light scattering properties of SALC thin film waveguide have been studied both theoretically and experimentally [24]. The scattering losses in smectic waveguides caused by dynamic distortions of the smectic layer planes are several orders of magnitude lower than in nematic waveguides [2,24], and SLCs may be used in nonlinear optical applications [2]. Recently, the reconfigurable smectic layer curvature has been studied [25]. The using of the external electric field to create the dynamic variations of the smectic layer configuration attracted a wide interest [25]. The different types of the periodic focal conic domain (FCD) arrays with the domain size, shape, orientation, and lattice symmetry controlled by external fields can be obtained [25].The applications of SALC such as soft-lithographic templates, superhydrophobic surfaces, microlens arrays, and optically selective photomasks have been developed [25].

The nonlinear optical phenomena in SALC have been investigated theoretically [26-36]. It has been shown that the light self-focusing, self-trapping, Brillouin-like SLS, and four-wave mixing (FWM) related to the light enhanced smectic layer normal displacement $u(\vec{r}, t)$ occur in SALC under certain conditions. The nonlinear effects based on this nonlinearity mechanism specific for SALC are strongly anisotropic, and the corresponding SLS gain coefficient is significantly larger than the one in the case of the Brillouin SLS in isotropic organic liquids. The nonlinear interaction of the surface plasmon polaritons (SPPs) in the metal-insulator-metal (MIM) waveguide has been analyzed [36]. In particular, it has been shown theoretically that the strong SLS of the transverse magnetic (TM) even modes can occur in the optical slab waveguide with a SALC core [35].

In this paper, we investigated in detail SLS in the Silicon-SALC slab waveguide. We discussed in detail the peculiarities of different types of LCs and concentrated on the optical properties of SALC. We derived the SALC layer equation of motion and the truncated equations for the optical wave slowly varying amplitudes (SVAs). We discussed the contribution of the TM even and odd modes and the transverse electric (TE) modes of the Silicon-SALC waveguide. We solved simultaneously the Maxwell equations including the nonlinear polarization for the waveguide modes and the equation of motion for the smectic layer normal displacement $u(\vec{r}, t)$ in the optical wave field. We evaluated $u(\vec{r}, t)$ and the hydrodynamic velocity $\vec{v}(\vec{r}, t)$ in the SALC core of the waveguide. We obtained the novel explicit solutions for the SVAs of the interfering waveguide modes and made numerical estimations of the waveguide mode parameters and the gain. The results of the numerical estimations are presented in Figures 2-8. The paper is constructed as follows. The theoretical model is presented in Section 2. The nonlinear polarization in the waveguide SALC core is evaluated in Section 3. The SVAs of the pumping and signal TM waveguide modes and the hydrodynamic velocity of smectic layers are calculated in Section 4. The conclusions are presented in Section 5.

\section{Theoretical Model}

A typical LC slab waveguide represents a LC thin film with a thickness of about $1 \mu \mathrm{m}$ sandwiched between two glass slides of lower refractive index than LC [2]. One of slides is covered with an organic film. The input laser radiation is inserted into the film via the coupling prism [2]. The laser excites the TE and/or TM modes in the film which are then introduced into the LC core [2]. Such a structure can be placed on a Si substrate [8]. One of the claddings can be made of $\mathrm{SiO}_{2}$ [8]. For the sake of definiteness, we consider the homeotropically oriented SALC core where the molecular elongated axes are perpendicular to the waveguide claddings and the smectic layer planes parallel to them. The structure of the optical slab waveguide with a homeotropically oriented SALC core is shown in Figure 1. 


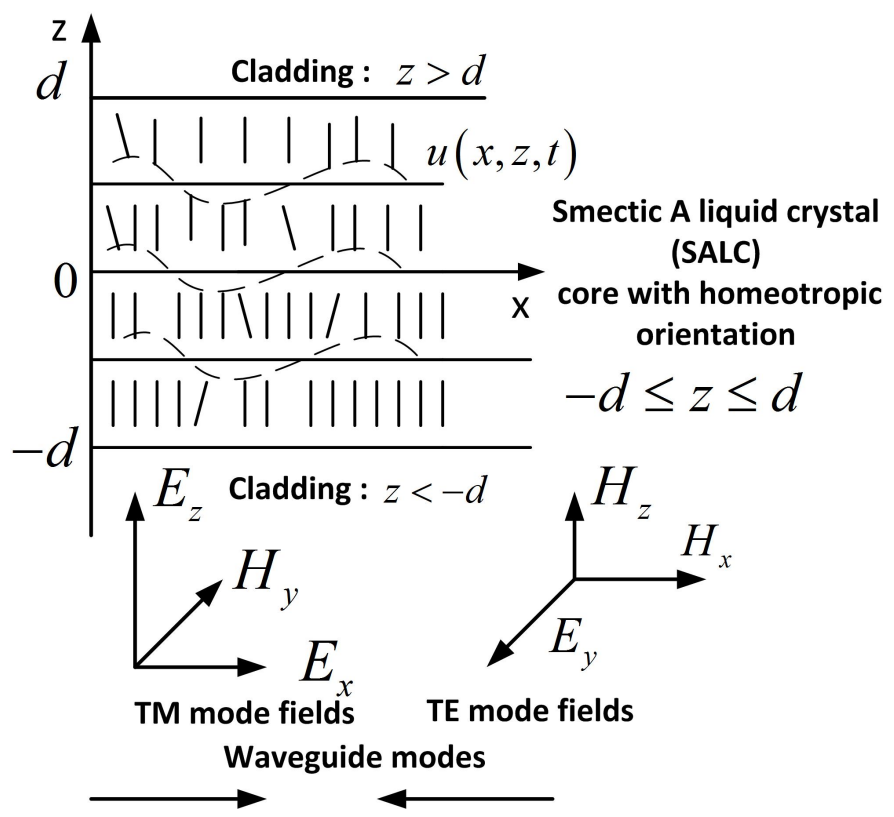

Figure 1. Optical slab waveguide with the homeotropically oriented SALC core of the thickness $2 d$. $E_{x, z}, H_{y}$ and $E_{y}, H_{x, z}$ are the electric and magnetic fields of the TM and TE modes, respectively.

Optical waves interact through the nonlinear polarization in a medium [37]. Generally, different types of SLS are described by the coupled wave equations for the light waves and for the corresponding material excitations [37]. The wave equation for electric field $\vec{E}(\vec{r}, t)$ of the optical wave propagating in a nonlinear medium has the form [37].

$$
\operatorname{curl} \operatorname{curl} \vec{E}+\mu_{0} \frac{\partial^{2} \vec{D}^{L}}{\partial t^{2}}=-\mu_{0} \frac{\partial^{2} \vec{D}^{N L}}{\partial t^{2}}
$$

where $\mu_{0}$ is the free space permeability, $\vec{D}^{L}$ and $\vec{D}^{N L}$ are the linear and nonlinear parts of the electric induction, respectively.

The SLS in the liquid crystalline waveguide with a SALC core is described by the coupled wave equations of the type (1) for the waveguide modes and the hydrodynamic equations for SALC. The SALC hydrodynamics in general case is very complicated taking into account the anisotropy and including the fluctuations of the mass density $\rho$, the layer displacement $u(\vec{r}, t)$ along the $Z$ axis normal to the layers and the change of the director $\vec{n}[15,16]$. The character of the fluctuation modes is determined by the propagation direction [15-18]. We assume that the SALC temperature is far from the temperature $T_{S m A-N}$ of the SALC-NLC phase transition. Since the optical losses in SALC are negligible [2] the waveguide temperature is assumed to be constant and the smectic A phase is stable. In such a case, the system of hydrodynamic equations for SALC has the form [15].

$$
\begin{gathered}
\rho \frac{\partial v_{i}}{\partial t}=-\frac{\partial \Pi}{\partial x_{i}}+\Lambda_{i}+\frac{\partial \sigma_{i k}^{\prime}}{\partial x_{k}} \\
\Lambda_{i}=-\frac{\delta F}{\delta u_{i}} \\
\sigma_{i k}^{\prime}=\alpha_{0} \delta_{i k} A_{l l}+\alpha_{1} \delta_{i z} A_{z z}+\alpha_{4} A_{i k}+\alpha_{56}\left(\delta_{i z} A_{z k}+\delta_{k z} A_{z i}\right)+\alpha_{7} \delta_{i z} \delta_{k z} A_{l l} \\
A_{i k}=\frac{1}{2}\left(\frac{\partial v_{i}}{\partial x_{k}}+\frac{\partial v_{k}}{\partial x_{i}}\right) \\
\operatorname{div} \vec{v}=0
\end{gathered}
$$




$$
\begin{gathered}
v_{z}=\frac{\partial u}{\partial t} \\
\delta_{i k}= \begin{cases}1, & i=k \\
0, & i \neq k\end{cases}
\end{gathered}
$$

where $\vec{v}$ is the hydrodynamic velocity, $\Pi$ is the pressure, $\vec{\Lambda}$ is the generalized force density, $\sigma_{i k}^{\prime}$ is the viscous stress tensor, $\alpha_{i}$ are the viscosity Leslie coefficients, $F$ is the free energy density of SALC. The SALC free energy density in the presence of the external electric field $\vec{E}(\vec{r}, t)$ has the form.

$$
F=\frac{1}{2} B\left(\frac{\partial u}{\partial z}\right)^{2}+\frac{1}{2} K\left(\frac{\partial^{2} u}{\partial x^{2}}+\frac{\partial^{2} u}{\partial y^{2}}\right)^{2}-\frac{1}{2} \varepsilon_{0} \varepsilon_{i k} E_{i} E_{k}
$$

Here $B \sim 10^{6}-10^{7} \mathrm{~J} / \mathrm{m}^{3}$ is the elastic constant related to the layer compression, $K \sim 10^{-11} \mathrm{~N}$ is the Frank elastic constant associated with the SALC purely orientational energy, $\varepsilon_{0}$ is the free space permittivity, and $\varepsilon_{i k}$ is the SALC permittivity tensor including the nonlinear terms related to the smectic layer strains. SALC is an optically uniaxial medium with the optical axis $Z$ normal to the layer plane. It is given by [16].

$$
\begin{gathered}
\varepsilon_{x x}=\varepsilon_{y y}=\varepsilon_{\perp}+a_{\perp} \frac{\partial u}{\partial z} \\
\varepsilon_{z z}=\varepsilon_{\|}+a_{\|} \frac{\partial u}{\partial z} \\
\varepsilon_{x z}=\varepsilon_{z x}=-\varepsilon_{a} \frac{\partial u}{\partial x}, \varepsilon_{y z}=\varepsilon_{z y}=-\varepsilon_{a} \frac{\partial u}{\partial y}
\end{gathered}
$$

where $\varepsilon_{\perp}, \varepsilon_{\|}$are the diagonal components of the permittivity tensor perpendicular and parallel to the optical axis, respectively, $a_{\perp} \sim 1, a_{\|} \sim 1$ are the phenomenological dimensionless coefficients, and $\varepsilon_{a}$ is the permittivity anisotropy. In our case, the losses in SALC can be neglected and the linear permittivity is real [2].

$$
\varepsilon_{a}=\varepsilon_{\|}-\varepsilon_{\perp}
$$

For the wave vector $\vec{k}_{S}$ oblique to the smectic layer plane in SALC there exist two practically uncoupled acoustic modes. One of these modes is the ordinary longitudinal sound wave caused by the mass density oscillations and described by the dispersion relation $\Omega=s_{1} k_{S}$ independent of the propagation direction where the sound velocity $s_{1}=\sqrt{A / \rho}$, and $A$ is the elastic constant related to bulk compression [15-18]. The second mode is the so-called second sound (SS) with the following dispersion relation $[15,17]$.

$$
\Omega_{S S}=s_{2} \frac{k_{S \perp} k_{S z}}{k_{S}}, s_{2}=\sqrt{\frac{B}{\rho}}
$$

where $s_{2}$ is the SS velocity, $k_{S \perp}, k_{S z}$ are the SS wave vector components in the layer plane and normal to it, respectively. SS corresponds to the changes in the layer spacing, it is neither longitudinal, nor transverse, and vanishes for the wave vector parallel or perpendicular to the smectic layer plane as it is seen from Equation (14) [15-18]. Since the elastic constant $B \ll A \sim 10^{9} \mathrm{~J} / \mathrm{m}^{3}$, the SS may propagate in the SALC without the density change [15-18]. SS has been observed experimentally [19-21]. In such a case, SALC may be considered to be incompressible liquid according to Equation (6), the pressure $\Pi=0$, and the SALC energy density $F$ determined by Equation (9) does not include the bulk compression term. The purely orientational term second term in Equation (9) can be neglected since for the typical values of the elastic constants and $k_{S} \sim 10^{5} \mathrm{~m}^{-1} B \gg K k_{S}^{2}$. The normal layer displacement $u(\vec{r}, t)$ by definition has only one component along the $Z$ axis. Hence, the generalized force density $\vec{\Lambda}$ has only the $z$ component according to Equation (3): $\vec{\Lambda}=\left(0,0, \Lambda_{z}\right)$. Equation (7) is specific for SALC since it determines the condition of the smectic layer continuity and the absence of the permeation process which can be neglected in the high frequency limit $[15,17]$. 
Taking into account the assumptions mentioned above and combining Equations (2)-(12) we obtain the equation of motion for smectic layer normal displacement $u(\vec{r}, t)$ in an external electric field $\vec{E}(\vec{r}, t)[36]$.

$$
\begin{gathered}
-\rho \nabla^{2} \frac{\partial^{2} u}{\partial t^{2}}+\left[\alpha_{1} \nabla_{\perp}^{2} \frac{\partial^{2}}{\partial z^{2}}+\frac{1}{2}\left(\alpha_{4}+\alpha_{56}\right) \nabla^{2} \nabla^{2}\right] \frac{\partial u}{\partial t}+B \nabla_{\perp}^{2} \frac{\partial^{2} u}{\partial z^{2}} \\
=\frac{\varepsilon_{0}}{2} \nabla_{\perp}^{2}\left\{-2 \varepsilon_{a}\left[\frac{\partial}{\partial x}\left(E_{x} E_{z}\right)+\frac{\partial}{\partial y}\left(E_{y} E_{z}\right)\right]+\frac{\partial}{\partial z}\left[a_{\perp}\left(E_{x}^{2}+E_{y}^{2}\right)+a_{\|} E_{z}^{2}\right]\right\}
\end{gathered}
$$

where $\nabla_{\perp}^{2}=\left(\partial^{2} / \partial x^{2}\right)+\left(\partial^{2} / \partial y^{2}\right)$. Taking into account the SALC symmetry we may choose without the loss of generality the propagation plane in a slab waveguide as the $x z$ plane. Then, using expressions (10)-(12) we obtain for the linear and nonlinear parts of the electric induction $\vec{D}^{L}$ and $\vec{D}^{N L}$.

$$
\begin{gathered}
D_{x, y}^{L}=\varepsilon_{0} \varepsilon_{\perp} E_{x, y}, D_{z}^{L}=\varepsilon_{0} \varepsilon_{\|} E_{z} \\
D_{x}^{N L}=\varepsilon_{0}\left(a_{\perp} \frac{\partial u}{\partial z} E_{x}-\varepsilon_{a} \frac{\partial u}{\partial x} E_{z}\right) ; D_{y}^{N L}=\varepsilon_{0} a_{\perp} \frac{\partial u}{\partial z} E_{y} \\
D_{z}^{N L}=\varepsilon_{0}\left(a_{\|} \frac{\partial u}{\partial z} E_{z}-\varepsilon_{a} \frac{\partial u}{\partial x} E_{x}\right)
\end{gathered}
$$

It is seen from Equations (17) and (18) that the nonlinear polarization in SALC is related to the smectic layer normal and tangential strain $\partial u / \partial z$ and $\partial u / \partial x$ as it was mentioned above [26-36]. We solve the wave Equation (1) according to the SVA approximation procedure [37]. In the linear approximation, we solve the homogeneous part of Equation (1) neglecting the nonlinear polarization (17) and (18).

$$
\operatorname{curl} \operatorname{curl} \vec{E}+\mu_{0} \frac{\partial^{2} \vec{D}^{L}}{\partial t^{2}}=0
$$

We obtain from Equation (19) the general solution and the linear dispersion relations for the waveguide modes [38,39]. Then, we evaluate the nonlinear polarization (17) and (18), derive the truncated equations for the SVAs of the waveguide mode electric fields in the SALC core and evaluate the complex SVA magnitudes and phases $[37,38]$. In the next section, we evaluate the waveguide modes and the nonlinear polarization defined by Equations (17) and (18).

\section{Nonlinear Polarization in the SALC Core of the Waveguide}

The TM and TE mode electric and magnetic fields have the form, respectively [38-40].

$$
\begin{aligned}
& \vec{H}_{T M}(x, z, t)=H_{y}(x, z, t) \mathbf{a}_{y} ; \vec{E}_{T M}(x, z, t)=\left(E_{x}(x, z, t), 0, E_{z}(x, z, t)\right) \\
& \vec{E}_{T E}(x, z, t)=E_{y}(x, z, t) \mathbf{a}_{y} ; \vec{H}_{T E}(x, z, t)=\left(H_{x}(x, z, t), 0, H_{z}(x, z, t)\right)
\end{aligned}
$$

We consider separately the TM and TE modes propagating in the slab optical waveguide with the SALC core because Equations (15)-(18) show that in the framework of the slab waveguide model TE and TM modes do not interact. We start with the analysis of the TM even modes. Assuming that the waveguide is symmetric with the identical claddings $z>d, z<-d$ characterized by the same permittivity $\varepsilon_{r 2}$ and the refraction index $n_{2}=\sqrt{\varepsilon_{r 2}}$, solving Equation (1) in the linear approximation and using the boundary conditions for the tangential components of the magnetic and electric field in the cladding $H_{y C}$ and $E_{x C}$ and in the SALC core $H_{y S A}$ and $E_{x S A}$, respectively [38-40].

$$
H_{y C}(z=d)=H_{y S A}(z=d) ; E_{x C}(z=d)=E_{x S A}(z=d)
$$


we obtain for the electric field $E_{x, z S A}, E_{x, z C}$ in the SALC core $|z| \leq d$ and in the cladding $z>d, z<-d$, respectively [35,39].

$$
\begin{gathered}
E_{x S A}=-i E_{0 z S A} \frac{k \varepsilon \|}{\beta \varepsilon_{\perp}} \sin k z \exp [i(\omega t-\beta x)] \\
E_{z S A}=-E_{0 z S A} \cos k z \exp [i(\omega t-\beta x)] \\
E_{x C}=\left\{\begin{array}{c}
i \frac{\alpha}{\beta} E_{0 z C} \exp (-\alpha z) \exp i(\omega t-\beta x), z>d \\
-i \frac{\alpha}{\beta} E_{0 z C} \exp (\alpha z) \exp i(\omega t-\beta x), z<-d
\end{array}\right. \\
E_{z C}=\left\{\begin{array}{l}
E_{0 z C} \exp (-\alpha z) \exp i(\omega t-\beta x), z>d \\
E_{0 z C} \exp (\alpha z) \exp i(\omega t-\beta x), z<-d
\end{array}\right.
\end{gathered}
$$

Here $\omega$ is the optical mode angular frequency, $\beta$ is the propagation constant, $k$ is the wave vector in the core, and $\alpha$ is the wavenumber in the cladding. They are given by

$$
\begin{gathered}
\beta=\sqrt{\varepsilon_{\|}\left[\left(\frac{\omega}{c}\right)^{2}-\frac{k^{2}}{\varepsilon_{\perp}}\right]} \\
\alpha=\sqrt{\beta^{2}-\frac{\omega^{2}}{c^{2}} \varepsilon_{r 2}}
\end{gathered}
$$

Expression (27) shows that the TM mode propagates in an anisotropic medium as an extraordinary wave [41]. The wave vector $k$ for the TM even modes is defined by the dispersion relation

$$
\tan k d=\frac{\varepsilon_{\perp}}{\varepsilon_{r 2}} \sqrt{\left(\frac{V}{k d}\right)^{2}-\frac{\varepsilon_{\|}}{\varepsilon_{\perp}}} ; V=\frac{2 \pi d}{\lambda_{0}} \sqrt{\varepsilon_{\|}-\varepsilon_{r 2}} ; \varepsilon_{\|}>\varepsilon_{r 2}
$$

where $\lambda_{0}=2 \pi c / \omega$ and $c$ are the free space wavelength and light velocity, respectively. Consider now the TM odd modes. In this case, the electric field components $E_{x, z S A}^{\text {odd }}$ in the SALC has the form [39].

$$
\begin{gathered}
E_{z S A}^{o d d}=E_{0 z S A}^{o d d} \sin k z \exp i(\omega t-\beta x) \\
E_{x S A}^{o d d}=-\frac{i k \varepsilon_{\|}}{\beta \varepsilon_{\perp}} E_{0 z S A}^{o d d} \cos k z \exp i(\omega t-\beta x)
\end{gathered}
$$

The boundary conditions (22) give the following dispersion relation for the TM odd modes.

$$
-\cot k d=\frac{\varepsilon_{\perp}}{\varepsilon_{r 2}} \sqrt{\left(\frac{V}{k d}\right)^{2}-\frac{\varepsilon_{\|}}{\varepsilon_{\perp}}}
$$

The solution of the dispersion relations (29) and (32) for the TM even and odd modes are presented in Figure 2a,b, respectively. It is seen from Figure 2a,b that for the frequency $\omega=5 \pi \times 10^{14} \mathrm{~s}^{-1}$ and for the typical values of the waveguide parameters there exist two even TM modes $\mathrm{TM}_{0,1}^{\text {even }}$ and one odd TM mode $\mathrm{TM}_{1}^{\text {odd }}$. The normalized wavenumber $k d$ and propagation constant $\beta d$ dependence on the optical wavelength $\lambda$ for the even modes $\mathrm{TM}_{0,1}^{\text {even }}$ and for the odd mode $\mathrm{TM}_{1}^{\text {odd }}$ are presented in Figure $3 \mathrm{a}, \mathrm{b}$, respectively. The normalized wavenumber in the cladding $\alpha d$ spectral dependence is shown in Figure 4. It is seen from Figure 4 that the fundamental even mode $\mathrm{TM}_{0}^{\text {even }}$ does not have a cutoff while the second even mode $\mathrm{TM}_{1}^{\text {even }}$ has a cutoff wavelength coinciding with the cutoff wavelength in Figure $3 a, b$, respectively. Comparison of Figures $3 a$ and 4 shows that in the wavelength region under consideration $k d>\pi / 2$, and $\alpha d \neq 0$ for the odd mode $\mathrm{TM}_{1}^{\text {odd }}$ [39]. The solutions of the dispersion relations (29) and (32) presented in Figure 3a,b show that for the waveguide SALC core thickness of $2 d=2 \mu \mathrm{m}$, the typical values of LC and cladding permittivity [8], and the wavelengths $\lambda_{0} \approx$ 1.4-1.55 $\mu \mathrm{m}$ important for optical communications the single mode regime occurs. We consider the 
interaction of the TM modes with the close optical frequencies $\omega_{1,2}$ such that the frequency shift $\Delta \omega=$ $\omega_{1}-\omega_{2} \sim 10^{8}-10^{9} \mathrm{~s}^{-1} \ll \omega_{1}$ which is typical for the light scattering in SALC $[15,19]$. The numerical estimations of the propagation constant $\beta$ and the wave vector $k$ according to Equations (27) and (29) show that for the frequency shifts $\Delta \omega \sim 10^{8}-10^{9} \mathrm{~s}^{-1}$ the values of $\beta$ and $k$ are practically the same for the TM modes with the close frequencies $\omega_{1,2}$. Consequently, the strong interaction occurs only for the counter-propagating TM modes. For the sake of definiteness, we consider the interaction of the TM even modes (23) and (24). Obviously, the nonlinear interaction of the TM odd modes would be practically the same. Using expressions (23) and (24) we can write for such TM even mode electric field [35].

$$
\vec{E}_{S A 1,2}=\frac{1}{2} E_{0 z S A 1,2}(x, t)\left\{-\vec{a}_{x} i \frac{k \varepsilon_{\|}}{\beta \varepsilon_{\perp}} \sin k z \mp \vec{a}_{z} \cos k z\right\} \exp \left[i\left(\omega_{1,2} t \mp \beta x\right)\right]+\text { c.c. }
$$

where c.c. stands for complex conjugate, and $\vec{a}_{x}, \vec{a}_{z}$ are the unit vectors of the $X$ and $Z$ axes, respectively. We assume that $E_{0 z S A 1,2}(x, t)=\left|E_{0 z S A 1,2}\right| \exp i \theta_{1,2}$ are the complex SVAs [37].

$$
\left|\frac{\partial^{2} E_{0 z S A 1,2}}{\partial x^{2}}\right| \ll\left|\beta \frac{\partial E_{0 z S A 1,2}}{\partial x}\right| ;\left|\frac{\partial^{2} E_{0 z S A 1,2}}{\partial t^{2}}\right| \ll\left|\omega \frac{\partial E_{0 z S A 1,2}}{\partial t}\right|
$$
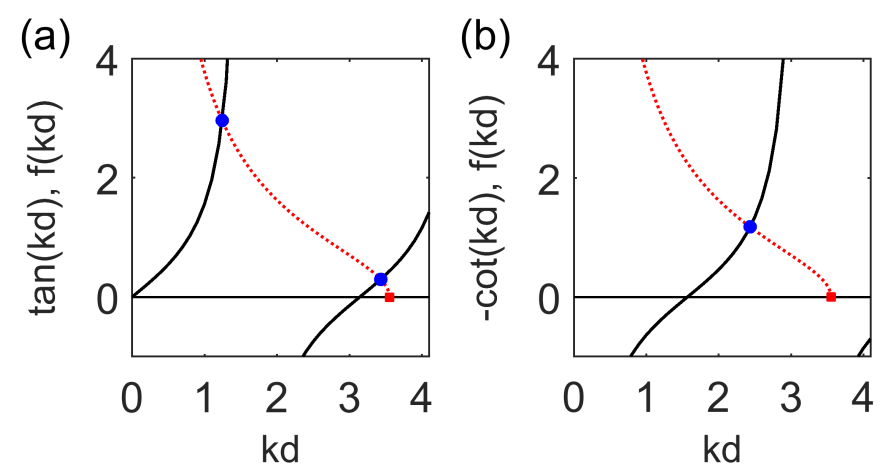

Figure 2. Graphic solution of the disperison relations for the TM even modes (a) and odd modes (b); $f(k d)=\frac{\varepsilon_{\perp}}{\varepsilon_{r 2}} \sqrt{\left(\frac{V}{k d}\right)^{2}-\frac{\varepsilon_{\|}}{\varepsilon_{\perp}}}, \omega=5 \pi \times 10^{14} S^{-1}$.

(a)

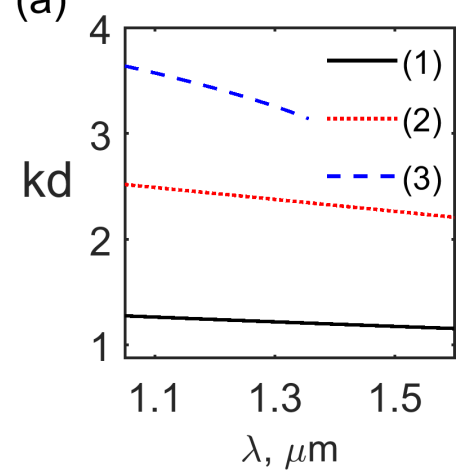

(b)

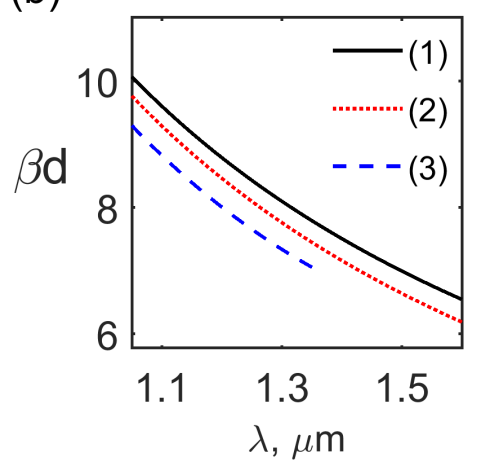

Figure 3. The normalized wavenumber $k d$ (a) and propagation constant $\beta d$ (b) dependence on the optical wavelength $\lambda$ for the even modes $\mathrm{TM}_{0,1}^{\text {even }}$ (curves 1,3 ) and the odd mode $\mathrm{TM}_{1}^{\text {odd }}$ (curve 2). 


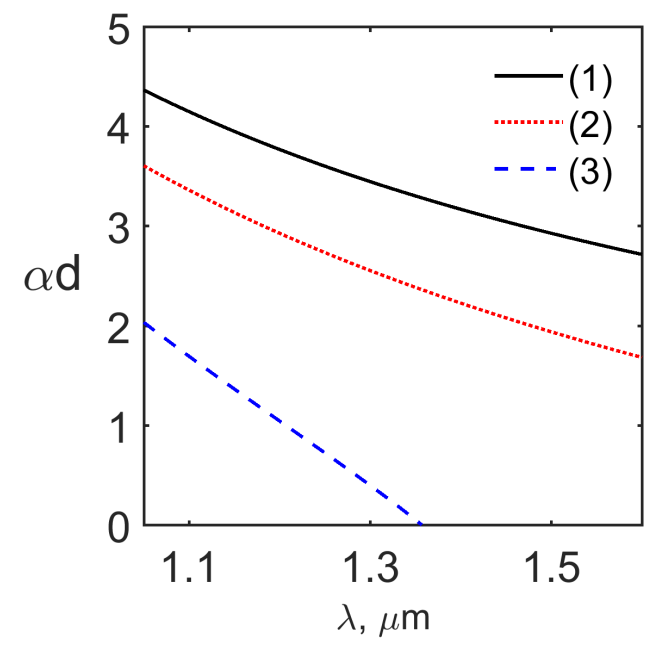

Figure 4. The dependence of normalized wavenumber in the cladding $\alpha d$ for the even modes $\mathrm{TM}_{0,1}^{\text {even }}$ (curves 1,3 ) and odd mode $\mathrm{TM}_{1}^{\text {odd }}$ (curve 2) on the optical wavelength.

At the small distances of several $\mathrm{mm}$ typical for the optical waveguide length the dependence of SVAs on $x$ and the dispersion effects can be neglected, and the SVAs $E_{0 z S A 1,2}(t)$ depend only on time. Substituting expressions (33) into equation of motion (15) and keeping in the right-hand side (RHS) only the terms with the frequency difference $\Delta \omega$ we obtain.

$$
\begin{gathered}
-\rho \nabla^{2} \frac{\partial^{2} u}{\partial t^{2}}+\left[\alpha_{1} \frac{\partial^{2}}{\partial x^{2}} \frac{\partial^{2}}{\partial z^{2}}+\frac{1}{2}\left(\alpha_{4}+\alpha_{56}\right) \nabla^{2} \nabla^{2}\right] \frac{\partial u}{\partial t}+B \frac{\partial^{2}}{\partial x^{2}} \frac{\partial^{2} u}{\partial z^{2}} \\
=-2 \varepsilon_{0} \beta^{2} k E_{0 z S A 1} E_{0 z S A 2}^{*}\left[\varepsilon_{a} \frac{\varepsilon_{\|}}{\varepsilon_{\perp}}+a_{\perp} \frac{1}{2}\left(\frac{k \varepsilon_{\|}}{\beta \varepsilon_{\perp}}\right)^{2}+\frac{1}{2} a_{\|}\right] \\
\times \sin 2 k z \exp \left\{i\left[\left(\omega_{1}-\omega_{2}\right) t-2 \beta x\right]\right\}+\text { c.c. }
\end{gathered}
$$

Then the particular solution of Equation (35) related to its RHS yields the dynamic grating of the smectic layer normal displacement.

$$
u(x, z, t)=U_{0} \sin 2 k z \exp \left\{i\left[\left(\omega_{1}-\omega_{2}\right) t-2 \beta x\right]\right\}+\text { c.c. }
$$

where

$$
\begin{gathered}
U_{0}=\frac{\varepsilon_{0} \beta^{2} k E_{0 z S A 1} E_{0 z S A 2}^{*}\left[\varepsilon_{a} \frac{\varepsilon_{\|}}{\varepsilon_{\perp}}+a_{\perp} \frac{1}{2}\left(\frac{k \varepsilon_{\|}}{\beta \varepsilon_{\perp}}\right)^{2}+\frac{1}{2} a_{\|}\right]}{2 \rho\left(\beta^{2}+k^{2}\right) G(k, \beta, \Delta \omega)} \\
\Gamma=\frac{1}{\rho}\left[4 \frac{\alpha_{1} \beta^{2} k^{2}}{\left(\beta^{2}+k^{2}\right)}+2\left(\alpha_{4}+\alpha_{56}\right)\left(\beta^{2}+k^{2}\right)\right] ; \Omega^{2}=4 \frac{B \beta^{2} k^{2}}{\rho\left(\beta^{2}+k^{2}\right)}
\end{gathered}
$$

Here $\Omega, \Gamma$ are SS frequency and decay factor, respectively [15-21]. The SS frequency $\Omega$ and decay factor $\Gamma$ dependence on the optical wavelength $\lambda$ for the first two TM modes are presented in Figure 5a,b, respectively. Numerical estimations show that for the typical values of SALC parameters [15-21], the optical wavelength in the range of $\lambda_{\text {opt }} \sim 1.3-1.55 \mu \mathrm{m}$ and $\Delta \omega \sim 10^{8}-10^{9} \mathrm{~s}^{-1}$ the homogeneous layer oscillations are overdamped. For this reason, the rapidly decaying homogeneous solution of Equation (35) can be neglected. We have taken into account only the solution (36) enhanced by the interfering optical TM modes (33). 

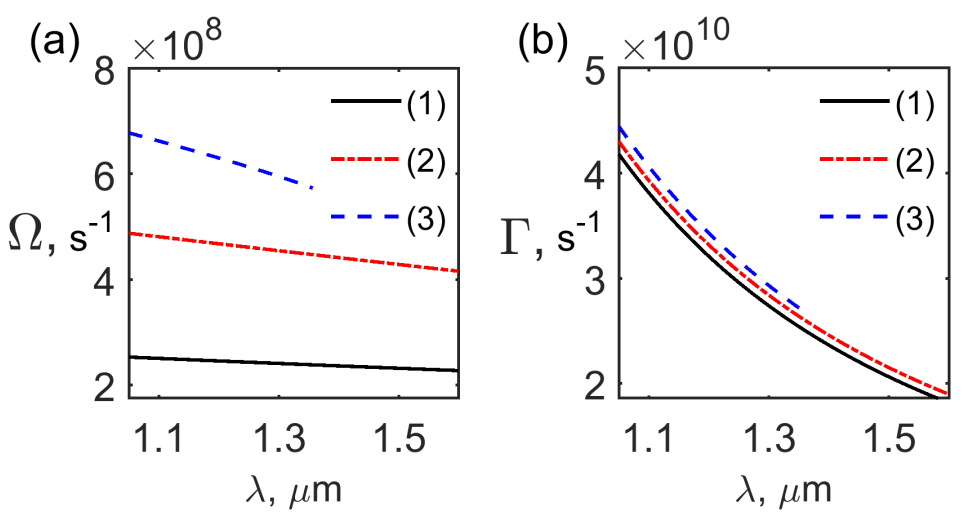

Figure 5. The SS frequency $\Omega$ (a) and decay constant $\Gamma$ (b) dependence on the optical wavelength for the even modes $\mathrm{TM}_{0,1}^{\text {even }}$ (curves 1,3) and the odd mode $\mathrm{TM}_{1}^{\text {odd }}$ (curve 2).

Substituting expressions (33) and (36) into Equations (17) and (18) we evaluate the nonlinear part of the electric induction $\vec{D}^{N L}=\left(D_{x}^{N L}, 0, D_{z}^{N L}\right)$ which has only $x$ and $z$ components for the TM modes. Separating the phase matched parts of $\vec{D}^{N L}$ with the frequencies $\omega_{1,2}$, respectively, we obtain.

$$
\begin{aligned}
& D_{x}^{N L}\left(\omega_{1}\right)=\varepsilon_{0} U_{0} i \beta E_{0 z S A 2} \sin k z \exp \left[i\left(\omega_{1} t-\beta x\right)\right] \\
& \times\left\{-a_{\perp} \frac{k^{2} \varepsilon_{\|}}{\beta^{2} \varepsilon_{\perp}} \cos 2 k z+2 \varepsilon_{a} \cos ^{2} k z\right\} \\
& D_{z}^{N L}\left(\omega_{1}\right)=\varepsilon_{0} k U_{0} E_{0 z S A 2} \cos k z \exp \left[i\left(\omega_{1} t-\beta x\right)\right] \\
& \times\left\{a_{\|} \cos 2 k z+2 \varepsilon_{a} \frac{\varepsilon_{\|}}{\varepsilon_{\perp}} \sin ^{2} k z\right\} \\
& D_{x}^{N L}\left(\omega_{2}\right)=\varepsilon_{0} U_{0}^{*} i E_{0 z S A 1} \sin k z \exp \left[i\left(\omega_{2} t+\beta x\right)\right] \\
& \times\left\{-a_{\perp} \frac{k^{2} \varepsilon_{\|}}{\beta \varepsilon_{\perp}} \cos 2 k z+2 \varepsilon_{a} \beta \cos ^{2} k z\right\} \\
& D_{z}^{N L}\left(\omega_{2}\right)=-\varepsilon_{0} k U_{0}^{*} E_{0 z S A 1} \cos k z \exp \left[i\left(\omega_{2} t+\beta x\right)\right] \\
& \times\left\{a_{\|} \cos 2 k z+2 \varepsilon_{a} \frac{\varepsilon_{\|}}{\varepsilon_{\perp}} \sin ^{2} k z\right\}
\end{aligned}
$$

The nonlinear polarization (40)-(43) is related to the specific cubic nonlinearity related to the smectic layer displacement which occurs without the change of the SALC mass density.

The electric field $E_{0 y S A 1,2}$ of the TE modes (21) is perpendicular to the optical axis Z. It has the form.

$$
\vec{E}_{S A 1,2}=\frac{1}{2} E_{0 y S A 1,2}(x, t) \vec{a}_{y} \cos k z \exp \left[i\left(\omega_{1,2} t \mp \beta x\right)\right]+\text { c.c. }
$$

TE modes propagate in an anisotropic medium as ordinary waves with the propagation constant $\beta^{2}=\left(\omega^{2} \varepsilon_{\perp} / c^{2}\right)-k^{2}$ including only the transverse permittivity $\varepsilon_{\perp}$ [41]. The boundary conditions for the TE modes have the form.

$$
E_{y C}(d)=E_{y S A}(d) ; H_{z C}(d)=H_{z S A}(d)
$$

They yield the TE mode dispersion relation similar to the isotropic medium [38].

$$
\tan k d=\sqrt{\frac{V_{T E}^{2}}{(k d)^{2}}-1} ; \quad V=\frac{2 \pi d}{\lambda_{0}} \sqrt{\varepsilon_{\perp}-\varepsilon_{r 2}}, \varepsilon_{\perp}>\varepsilon_{r 2}
$$


In LC typically $\varepsilon_{\|}>\varepsilon_{\perp}[2,14]$, and under the condition $\varepsilon_{\|}>\varepsilon_{r 2}>\varepsilon_{\perp}$ only TM modes can propagate in the slab optical waveguide. In general case, the nonlinear polarization enhanced by the TE modes includes only the component $D_{y}^{N L}=\varepsilon_{0} a_{\perp}(\partial u / \partial z) E_{y}$ as it is seen from expression (17), and the dynamic grating amplitude $U_{0 T E} \sim E_{0 y S A 1} E_{0 y S A 2}^{*}$. Obviously, the nonlinear interaction of the TE modes is isotropic and less pronounced than the TM mode interaction including both the longitudinal and the transverse component of the electric field. For this reason, we analyze in detail the TM mode nonlinear interaction.

\section{Evaluation of the TM Mode SVAs}

Using the standard procedure [37], we substitute expressions (33), (16), and (40)-(43) into Equation (1), separate the linear and nonlinear parts, neglect the small terms $\sim\left|\partial^{2} E_{0 z S A 1,2} / \partial t^{2}\right|$ according to the SVA approximation condition (34) and equate the phase matched terms the frequencies $\omega_{1,2}$, respectively. Then we obtain the coupled equations for the SVAs $E_{0 z S A 1,2}(t)$.

$$
\begin{gathered}
\varepsilon_{\|} \frac{\partial E_{0 z S A 1}}{\partial t}\left\{\mathbf{a}_{x} \frac{k}{\beta} \sin k z-\mathbf{a}_{z} i \cos k z\right\} \\
=\omega_{1} U_{0} E_{0 z S A 2}\left\{\mathbf{a}_{x} i \beta \sin k z\left[-a_{\perp} \frac{k^{2} \varepsilon_{\|}}{\beta^{2} \varepsilon_{\perp}} \cos 2 k z+2 \varepsilon_{a} \cos ^{2} k z\right]\right. \\
\left.+\mathbf{a}_{z} k \cos k z\left[a_{\|} \cos 2 k z+2 \varepsilon_{a} \frac{\varepsilon_{\|}}{\varepsilon_{\perp}} \sin ^{2} k z\right]\right\} \\
\varepsilon_{\|} \frac{\partial E_{0 z S A 2}}{\partial t}\left\{\mathbf{a}_{x} \frac{k}{\beta} \sin k z+\mathbf{a}_{z} i \cos k z\right\} \\
=\omega_{2} U_{0}^{*} E_{0 z S A 1}\left\{\mathbf{a}_{x} i \beta \sin k z\left[-a_{\perp} \frac{k^{2} \varepsilon_{\|}}{\beta^{2} \varepsilon_{\perp}} \cos 2 k z+2 \varepsilon_{a} \cos ^{2} k z\right]\right. \\
\left.-\mathbf{a}_{z} k \cos k z\left[a_{\|} \cos 2 k z+2 \varepsilon_{a} \frac{\varepsilon_{\|}}{\varepsilon_{\perp}} \sin ^{2} k z\right]\right\}
\end{gathered}
$$

We multiply Equations (46) and (47) by the vectors $\left\{\mathbf{a}_{x} \frac{k}{\beta} \sin k z-\mathbf{a}_{z} i \cos k z\right\}^{*}$ and $\left\{\mathbf{a}_{x} \frac{k}{\beta} \sin k z+\mathbf{a}_{z} i \cos k z\right\}^{*}$, respectively, substitute the SVA expressions

$$
E_{0 z S A 1,2}(x, t)=\left|E_{0 z S A 1,2}\right| \exp i \theta_{1,2}
$$

and separate the real and imaginary parts of the resulting equations. Then we obtain the following equations for the magnitudes $\left|E_{0 z S A 1,2}\right|$ and phases $\theta_{1,2}$ of the TM mode SVAs.

$$
\begin{gathered}
\frac{1}{\omega_{1}} \frac{\partial\left|E_{0 z S A 1}\right|^{2}}{\partial t} F_{1}(z)=\frac{\varepsilon_{0}\left|E_{0 z S A 1}\right|^{2}\left|E_{0 z S A 2}\right|^{2} \beta^{2} k^{2} h \operatorname{Im} G(k, \beta, \Delta \omega)}{\varepsilon_{\|} \rho\left(\beta^{2}+k^{2}\right)|G(k, \beta, \Delta \omega)|^{2}} F_{2}(z) \\
\frac{1}{\omega_{2}} \frac{\partial\left|E_{0 z S A 2}\right|^{2}}{\partial t} F_{1}(z)=-\frac{\varepsilon_{0}\left|E_{0 z S A 1}\right|^{2}\left|E_{0 z S A 2}\right|^{2} \beta^{2} k^{2} h \operatorname{Im} G(k, \beta, \Delta \omega)}{\varepsilon_{\|} \rho\left(\beta^{2}+k^{2}\right)|G(k, \beta, \Delta \omega)|^{2}} F_{2}(z) \\
\varepsilon_{\|} \frac{\partial \theta_{1}}{\partial t} F_{1}(z)=\omega_{1} \varepsilon_{0}\left|E_{0 z S A 2}\right|^{2} \frac{\beta^{2} k^{2} h \operatorname{Re} G(k, \beta, \Delta \omega)}{2 \rho\left(\beta^{2}+k^{2}\right)|G(k, \beta, \Delta \omega)|^{2}} F_{2}(z) \\
\varepsilon_{\|} \frac{\partial \theta_{2}}{\partial t} F_{1}(z)=\omega_{2} \varepsilon_{0} \frac{\beta^{2} k^{2}\left|E_{0 z S A 1}\right|^{2} h \operatorname{Re} G(k, \beta, \Delta \omega)}{2 \rho\left(\beta^{2}+k^{2}\right)|G(k, \beta, \Delta \omega)|^{2}} F_{2}(z)
\end{gathered}
$$


where

$$
\begin{aligned}
F_{1}(z)=\left(\frac{k}{\beta}\right)^{2} \sin ^{2} k z & +\cos ^{2} k z ; \quad F_{2}(z)=\left\{\sin ^{2} k z\left[-a_{\perp} \frac{k^{2} \varepsilon_{\|}}{\beta^{2} \varepsilon_{\perp}} \cos 2 k z+2 \varepsilon_{a} \cos ^{2} k z\right]\right. \\
& \left.+\cos ^{2} k z\left[a_{\|} \cos 2 k z+2 \varepsilon_{a} \frac{\varepsilon_{\|}}{\varepsilon_{\perp}} \sin ^{2} k z\right]\right\}
\end{aligned}
$$

and

$$
h=\varepsilon_{a} \frac{\varepsilon_{\|}}{\varepsilon_{\perp}}+a_{\perp} \frac{1}{2}\left(\frac{k \varepsilon_{\|}}{\beta \varepsilon_{\perp}}\right)^{2}+\frac{1}{2} a_{\|}
$$

Combining Equations (49) and (50) we obtain for the SVA magnitudes $\left|E_{0 z S A 1,2}\right|$

$$
\frac{\partial}{\partial t}\left(\frac{\left|E_{0 z S A 1}\right|^{2}}{\omega_{1}}+\frac{\left|E_{0 z S A 2}\right|^{2}}{\omega_{2}}\right)=0
$$

and

$$
\frac{\left|E_{0 z S A 1}\right|^{2}}{\omega_{1}}+\frac{\left|E_{0 z S A 2}\right|^{2}}{\omega_{2}}=\text { const }=I_{0}
$$

where

$$
I_{0}=\frac{\left|E_{0 z S A 1}(0)\right|^{2}}{\omega_{1}}+\frac{\left|E_{0 z S A 2}(0)\right|^{2}}{\omega_{2}}
$$

Equation (56) is the Manley-Rowe relation for the SVA magnitudes $\left|E_{0 z S A 1,2}\right|$ which corresponds to the conservation of the photon number in the SLS process [37]. It is seen from Equation (38) that for $\Delta \omega=\omega_{1}-\omega_{2}>0$ the imaginary part $\operatorname{Im} G(k, \beta, \Delta \omega)<0$, and the intensity $\left|E_{0 z S A 1}\right|^{2}$ of the TM mode with the higher frequency $\omega_{1}$ is decreasing with time while the intensity $\left|E_{0 z S A 2}\right|^{2}$ of the TM mode with the lower frequency $\omega_{2}$ is increasing. Consequently, the TM modes with the frequencies $\omega_{1,2}$ are the pumping and signal waves, respectively, and the Stokes type SLS occurs [37]. Equations (49) and (50) describe the energy exchange between the TM modes, while Equations (51) and (52) describe the cross-phase modulation (XPM) process.

We introduce the dimensionless variables

$$
I_{1,2}=\frac{\left|E_{0 z S A 1,2}\right|^{2}}{\omega_{1,2} I_{0}}
$$

such that $I_{1}+I_{2}=1$. Substituting expressions (58) into Equations (49) and (50), integrating both parts of these equations over $z$ from $-d$ up to $d$ and using the Manley-Rowe relation (56) we obtain the following solutions for the normalized SVA intensities $I_{1,2}$.

$$
\begin{aligned}
& I_{1}(t)=\frac{I_{1}(0)}{\left\{\left(1-I_{1}(0)\right) \exp [g F(k d) t]+I_{1}(0)\right\}} \\
& I_{2}(t)=\frac{\left(1-I_{1}(0)\right)}{\left(1-I_{1}(0)\right)+I_{1}(0) \exp [-g F(k d) t]}
\end{aligned}
$$

where the gain $g$ and the geometric factor $F(k d)$ are given by.

$$
g=\frac{\varepsilon_{0}}{\varepsilon_{\|}} \frac{\omega_{1} \omega_{2} I_{0} \beta^{2} k^{2} h|\operatorname{Im} G(k, \beta, \Delta \omega)|}{\rho\left(\beta^{2}+k^{2}\right)|G(k, \beta, \Delta \omega)|^{2}}>0
$$




$$
\begin{gathered}
F(k d)=\left\{\left[a_{\perp} \frac{k^{2} \varepsilon_{\|}}{\beta^{2} \varepsilon_{\perp}}+\varepsilon_{a}\left(1+\frac{\varepsilon_{\|}}{\varepsilon_{\perp}}\right)+a_{\|}\right] k d+\left[-a_{\perp} \frac{k^{2} \varepsilon_{\|}}{\beta^{2} \varepsilon_{\perp}}+a_{\|}\right] \sin 2 k d\right. \\
\left.+\frac{1}{4}\left[a_{\perp} \frac{k^{2} \varepsilon_{\|}}{\beta^{2} \varepsilon_{\perp}}-\varepsilon_{a}\left(1+\frac{\varepsilon_{\|}}{\varepsilon_{\perp}}\right)+a_{\|}\right] \sin 4 k d\right\}\left\{\left[\left(\frac{k}{\beta}\right)^{2}+1\right] 2 k d+\left[1-\left(\frac{k}{\beta}\right)^{2}\right] \sin 2 k d\right\}^{-1}
\end{gathered}
$$

The pumping intensity threshold in the SLS process described by expressions (59) and (60) is absent since the losses in SALC can be neglected as it was mentioned above. Comparison of expressions (59) and (60) shows that for the initial pumping wave intensity larger than the initial signal wave intensity $I_{1}(0)>I_{2}(0)$ the crossing time $t_{0}>0$ exists where $I_{1}\left(t_{0}\right)=I_{2}\left(t_{0}\right)$. It is given by.

$$
t_{0}=\frac{1}{g F(k d)} \ln \left[\frac{I_{1}(0)}{I_{2}(0)}\right]
$$

Substituting expression (63) into expressions (59) and (60) we obtain.

$$
I_{1,2}(t)=\frac{1}{2}\left\{1 \mp \tanh \left[\frac{1}{2} g F(k d)\left(t-t_{0}\right)\right]\right\}
$$

The spectral dependence of the gain $g$ and its dependence on the normalized intensity $I_{0}$ are presented in Figure 6a,b.
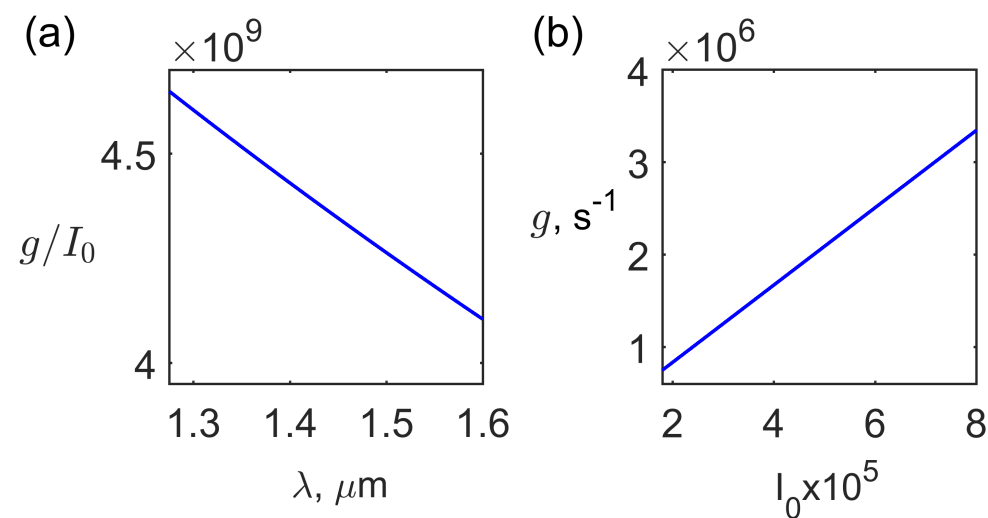

Figure 6. The normalized gain $g / I_{0}\left(\mathrm{~s}^{-2} \mathrm{~V}^{-2} \mathrm{~m}^{2}\right)$ dependence on the optical wavelength $\lambda$ (a); the gain $g$ dependence on the normalized intensity $I_{0}\left(\mathrm{~V}^{2} \mathrm{~m}^{-2} \mathrm{~s}\right)$ for the optical wavelength $\lambda=1.55 \mu \mathrm{m}(\mathbf{b})$.

Figure 6a shows that the gain is slightly varying in the optical wavelength range of interest because $\Gamma \gg \Omega$ as it is seen from Figure $5 \mathrm{a}, \mathrm{b}$. The gain $g$ has a maximum value $g_{\max }$ at the SS resonance condition when $\Delta \omega=\Omega$ and $\operatorname{Re} G(k, \beta, \Delta \omega)=0$ according to expression (38). The numerical estimations show that for the typical values of $k, \beta \sim 10^{6} \mathrm{~m}^{-1}$ and $\Delta \omega \sim 10^{8}-10^{9} \mathrm{~s}^{-1}$ the SS resonance condition can be satisfied. The numerical estimations also show that for the values of $k d$ defined by the dispersion relation (29) $F(k d) \sim 1$. The dependence of the gain $g$ on the normalized intensity $I_{0}$ is linear as it is seen from Figure 6b. Such a dependence is typical for the Brillouin and Rayleigh SLS [37]. The SLS in our case is essentially orientational since the optical nonlinearity mechanism is related to the SALC layer displacement and occurs without the mass density change [15]. For the feasible optical wave electric fields $E$ the condition $\varepsilon_{0} E^{2} / B \ll 1$ is always valid, and the gain saturation does not take place.

It is seen from expressions (59) and (60) that for $t \rightarrow \infty$ the pumping wave intensity is depleted $I_{1}(t) \rightarrow 0$ while the signal wave intensity is amplified up to the saturation level $I_{2}(t) \rightarrow 1$. The time dependence for the normalized intensities $I_{1,2}(t)$ for the initial conditions $I_{1}(0)=0.8, I_{2}(0)=0.2$, pumping wavelength $\lambda_{1}=1.55 \mu \mathrm{m}$ and the pumping wave electric field amplitude $E_{0 z S A 1}=10^{5} \mathrm{~V} / \mathrm{m}$, $10^{6} \mathrm{~V} / \mathrm{m}$ is shown in Figure 7. It is seen from Figure $7 \mathrm{a}, \mathrm{b}$ that the amplified signal wave rise time is 
about $60 \mu \mathrm{sec}$ and $0.6 \mu \mathrm{sec}$ for the feasible electric field $\sim 10^{5} \mathrm{~V} / \mathrm{m}, 10^{6} \mathrm{~V} / \mathrm{m}$, respectively, which is much faster than the director axis relaxation time $\tau_{r} \sim 1 \mathrm{~ms}$ in NLC [14].

(a)

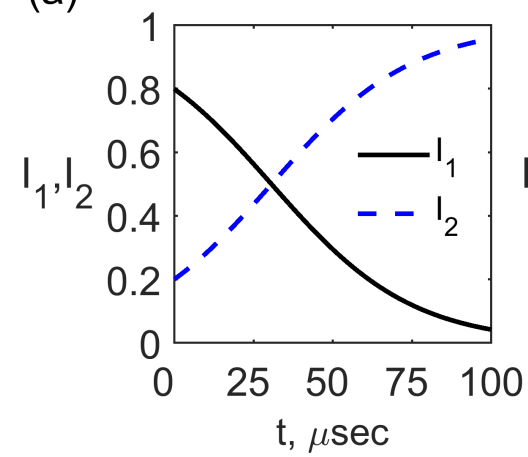

(b)

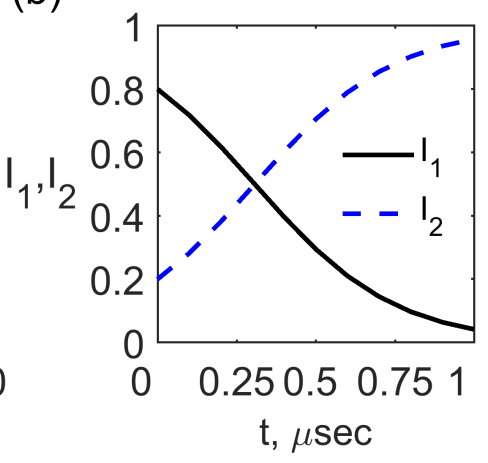

Figure 7. The time dependence of the normalized intensities $I_{1,2}(t)$ for the initial conditions $I_{1}(0)=0.8$, $I_{2}(0)=0.2$, pumping wavelength $\lambda_{1}=1.55 \mu \mathrm{m}$ and the pumping wave electric field amplitude $E_{0 z S A 1}=10^{5} \mathrm{~V} / \mathrm{m}(\mathbf{a})$ and $10^{6} \mathrm{~V} / \mathrm{m}(\mathbf{b})$.

Integrating both parts of Equations (51) and (52) over $z$ from $-d$ up to $d$ and substituting expressions (58)-(60), (62) into these equations, we obtain the expressions for the pumping and signal wave phases $\theta_{1,2}$. They have the form.

$$
\begin{gathered}
\theta_{1}(t)=\frac{\operatorname{Re} G(k, \beta, \Delta \omega)}{2|\operatorname{Im} G(k, \beta, \Delta \omega)|} \ln \left\{\exp [g F(k d) t]\left(1-I_{1}(0)\right)+I_{1}(0)\right\} \\
\theta_{2}(t)=-\frac{\operatorname{Re} G(k, \beta, \Delta \omega)}{2|\operatorname{Im} G(k, \beta, \Delta \omega)|} \ln \left\{I_{1}(0) \exp [-g F(k d) t]+\left(1-I_{1}(0)\right)\right\}
\end{gathered}
$$

It is seen from Equations (65) and (66) that XPM occurs, and the depletion of the pumping wave is accompanied by rapid linear increase of its phase $\theta_{1}(t)$ which corresponds to the fast oscillations of the amplitude $E_{0 z S A 1}$.

$$
t \rightarrow \infty, \theta_{1}(t) \approx \frac{\operatorname{Re} G(k, \beta, \Delta \omega)}{2|\operatorname{Im} G(k, \beta, \Delta \omega)|}(g F(k d) t) \rightarrow \infty
$$

The phase of the amplified signal wave $\theta_{2}(t)$ tends to the constant level:

$$
t \rightarrow \infty, \theta_{2}(t) \rightarrow-\frac{\operatorname{Re} G(k, \beta, \Delta \omega)}{2|\operatorname{Im} G(k, \beta, \Delta \omega)|} \ln I_{2}(0)
$$

The temporal evolution of $\cos \theta_{1,2}(t)$ is shown in Figure $8 \mathrm{a}, \mathrm{b}$, respectively. The characteristic time of the phase variation is about $10^{-4} \mathrm{~s}$ for the pumping wave electric field amplitude $E_{0 z S A 1}=10^{5} \mathrm{~V} / \mathrm{m}$. The comparison of expressions (38), (61), (65) and (66) shows that in the SS resonance case $\Delta \omega=\Omega$, $\operatorname{Re} G(k, \beta, \Delta \omega)=0, \mathrm{XPM}$ is absent: $\theta_{1,2}=$ const.

Consider now the hydrodynamic behavior of the SALC core. Substituting expressions (48), (58) and (64) into expression (37) we obtain the explicit expression of the smectic layer grating amplitude $U_{0}$.

$$
U_{0}=\frac{\varepsilon_{0} \beta^{2} k I_{0} \sqrt{\omega_{1} \omega_{2}}\left[\varepsilon_{a} \frac{\varepsilon_{\|}}{\varepsilon_{\perp}}+a_{\perp} \frac{1}{2}\left(\frac{k \varepsilon_{\|}}{\beta \varepsilon_{\perp}}\right)^{2}+\frac{1}{2} a_{\|}\right]}{4 \rho\left(\beta^{2}+k^{2}\right) G(k, \beta, \Delta \omega)} \frac{\exp i\left(\theta_{1}-\theta_{2}\right)}{\cosh \left[\frac{1}{2} g F(k d)\left(t-t_{0}\right)\right]}
$$


It is seen from Equation (69) that the crossing time $t_{0}$ corresponds to the maximum of the smectic layer strain pulse. Substituting expressions (36) and (69) into Equations (6) and (7) we obtain the following expressions of the hydrodynamic velocity components $v_{x, z}(x, z, t)$.

$$
\begin{aligned}
& v_{x}=\frac{k \Delta \omega}{\beta} U_{0} \cos 2 k z \exp i\left[\left(\omega_{1}-\omega_{2}\right) t-2 \beta x\right]+c . c . \\
& v_{z}=i \Delta \omega U_{0} \sin 2 k z \exp i\left[\left(\omega_{1}-\omega_{2}\right) t-2 \beta x\right]+c . c .
\end{aligned}
$$

It is seen from expressions (70) and (71) that they also have the form of the pulses (69).

(a)

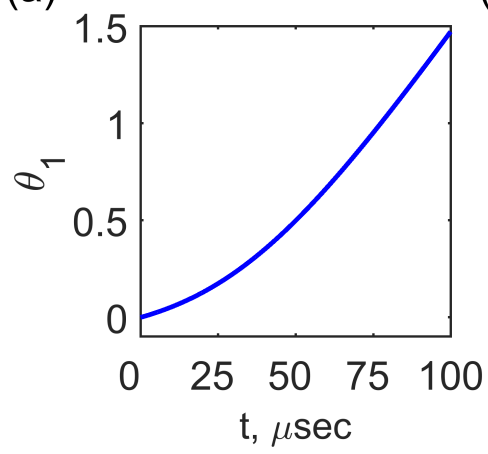

(b)

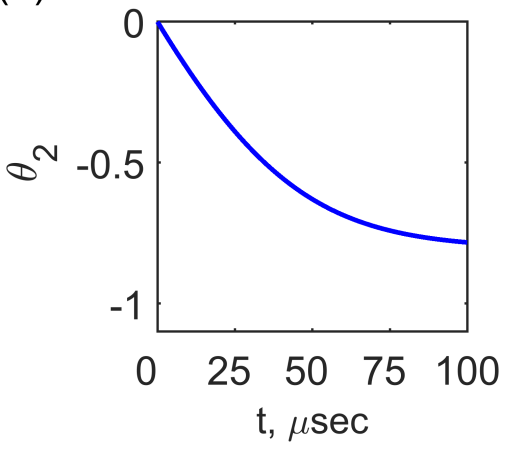

Figure 8. The temporal evolution of the phases $\theta_{1}(t)(\mathbf{a})$ and $\theta_{2}(t)(\mathbf{b})$ for the pumping wave electric field amplitude $E_{0 z S A 1}=10^{5} \mathrm{~V} / \mathrm{m}$ and the pumping wavelength $\lambda_{1}=1.33 \mu \mathrm{m}$.

\section{Conclusions}

We investigated theoretically the nonlinear optical phenomena in the optical slab waveguide with the SALC core. We calculated the TM and TE modes in such a strongly anisotropic waveguide. We have shown that the single mode regime can be realized for the waveguide core thickness of about $1-2 \mu \mathrm{m}$ and optical wavelength of $\lambda_{\text {opt }} \sim 1.35-1.55 \mu \mathrm{m}$ important for the optical communication applications. The cubic nonlinearity of SALC is related to the smectic layer normal displacement. The nonlinear interaction is especially strong for the counter-propagating TM modes. We solved simultaneously the equation of motion for the smectic layer normal displacement in the optical field and the wave equation for the TM mode electric field using SVA approximation. The interfering optical fields create the smectic layer displacement dynamic grating which propagates in SALC without the mass density change. As a result the nonlinear polarization occurs and the SLS accompanied by XPM takes place in the waveguide. We evaluated the pumping and signal TM mode SVA magnitudes and phases. In the resonance case when the TM mode frequency difference $\Delta \omega$ equals to the SS frequency $\Omega$ the gain $g$ has a maximum value, and XPM is absent. The smectic layer strain has a pulse form with a maximum corresponding to the crossing time of the pumping and signal TM modes. We also evaluated the hydrodynamic velocity enhanced by the interfering TM modes. The numerical estimations show that the SLS in SALC is much faster than the light scattering in NLC related to the director reorientation.

Author Contributions: Conceptualization, B.I.L.; Methodology, B.I.L. and Y.B.-E.; Validation, B.I.L., Y.B.-E., and D.I.; Formal Analysis, B.I.L. and D.I.; Investigation, B.I.L., Y.B.-E., and D.I.; Resources, Y.B.-E.; Writing-Original Draft Preparation, B.I.L.; Writing-Review and Editing, B.I.L., Y.B.-E., and D.I.; Visualization, D.I.; Supervision, Y.B.-E.; Project Administration, Y.B.-E.; Funding Acquisition, B.I.L. and Y.B.-E.

Funding: This research was funded by Holon Institute of Technology (HIT), Holon, Israel.

Conflicts of Interest: The authors declare no conflict of interest. 


\section{References}

1. Zografopoulos, D.C.; Asquini, R.; Kriezis, E.E.; d'Alessandro, A.; Beccherelli, R. Guided-wave liquid crystal photonics. Lab Chip 2012, 12, 3598-3610. [CrossRef]

2. Khoo, I.C. Liquid Crystals, 2nd ed.; Wiley: Hoboken, NJ, USA, 2007.

3. Lazarev, G.; Chen, Po.; Strauss, J.; Fontaine, N.; Forbes, A. Beyond the display: Phase-only liquid crystal on Silicon devices and their applications in photonics. Opt. Express 2019, 27, 16206-16249. [CrossRef] [PubMed]

4. Frisken, S.; Clarke, I.; Poole, S. Technology and applications of liquid crystal on Silicon (LCoS) in telecommunications. In Optical Fiber Telecommunications VIA, 6th ed.; Kaminov, I.P., Li, T., Willner, A.E., Eds.; Academic Press-Elsevier: Oxford, UK, 2013; pp. 709-742.

5. Li, M.; Zhang, L.; Tong, L.-M.; Dai, D.-X. Hybrid silicon nonlinear photonics. Photonics Res. 2018, 6, B13-B22. [CrossRef]

6. Whinnery, J.R.; Hu, C.; Kwon, Y.S. Liquid-crystal waveguides for integrated optics. IEEE J. Quantum Electron. 1977, 13, 262-267. [CrossRef]

7. d'Alessandro, A.; Bellini, B.; Donisi, D.; Beccherelli, R.; Asquini, R. Nematic liquid crystal optical channel waveguides on Silicon. IEEE J. Quantum Electron. 2006, 42, 1084-1090. [CrossRef]

8. Donisi, D.; Bellini, B.; Beccherelli, R.; Asquini, R.; Gilardi, G.; Trotta, M.; d'Alessandro, A. A switchable liquid-crystal optical channel waveguide on Silicon. IEEE J. Quantum Electron. 2010, 46, 762-768. [CrossRef]

9. Cos, J.; Ferré-Borrull, J.; Marsal, L.F. Tunable waveguides based on liquid-infiltrated silicon photonic crystals. Phys. Status Solidi C 2011, 8, 1075-1078. [CrossRef]

10. Cai, D.-P.; Pan, H.-Y.; Tsai, J.-F.; Chiu, H.-K.; Nian, S.-C.; Chang, S.H.; Chen, C.-C.; Lee, C.-C. Liquid crystal infiltrated waveguide with distributed Bragg reflectors. Opt. Mater. Express 2011, 1, 1471-1477. [CrossRef]

11. Huang, C.-C. Solving the full anisotropic liquid crystal waveguides by using an iterative pseudospectral-based eigenvalue method. Opt. Express 2011, 19, 3363-3378. [CrossRef]

12. Shenoy, M.R.; Sharma, M.; Sinha, A. An electrically controlled nematic liquid core waveguide with a low switching threshold. J. Light. Technol. 2015, 33, 1948-1953. [CrossRef]

13. d'Alessandro, A.; Martini, L.; Gilardi, G.; Beccherelli, R.; Asquini, R. Polarization-independent nematic liquid crystal waveguides for optofluidic applications. IEEE Photonic Technol. Lett. 2015, 27, 1709-1712. [CrossRef]

14. Khoo, I.C. Nonlinear optics of liquid crystalline materials. Phys. Rep. 2009, 471, 221-267. [CrossRef]

15. de Gennes, P.G.; Prost, J. The Physics of Liquid Crystals, 2nd ed.; Oxford University Press: New York, NY, USA, 1993.

16. Stephen, M.J.; Straley, J.P. Physics of Liquid Crystals. Rev. Mod. Phys. 1974, 46, 617-704. [CrossRef]

17. de Gennes, P.G. The Physics of Liquid Crystals; Clarendon Press-Oxford: Wotton-under-Edge, UK, 1974.

18. Chandrasekhar, S. Liquid Crystals, 2nd ed.; Cambridge University Press: Cambridge, UK, 1992.

19. Liao, Y.; Clark, N.A.; Pershan, P.S. Brillouin scattering from smectic liquid crystals. Phys. Rev. Lett. 1973, 30, 639-641. [CrossRef]

20. Ricard, L.; Prost, J. "Second sound" propagation and the smectic response function. J. Phys. Colloq. 1979, 40, C3-83-C3-86. [CrossRef]

21. Ricard, L.; Prost, J. Critical behavior of second sound near the smectic A nematic phase transition. J. Phys. 1981, 42, 861-873. [CrossRef]

22. Qian, S.; Iannacchione, G.S.; Finotello, D. Critical behavior of a smectic-A to nematic phase transition imbedded in a random network of voids. Phys. Rev. E 1998, 57, 4305-4315. [CrossRef]

23. Khoo, I.C. Extreme nonlinear optics of nematic liquid crystals. J. Opt. Soc. Am. B 2011, 28, A45-A55. [CrossRef]

24. Giallorenzi, T.G.; Weiss, J.A.; Sheridan, J.P. Light scattering from smectic liquid-crystal waveguides. J. Appl. Phys. 1976, 47, 1820-1826. [CrossRef]

25. Ma, L.-L.; Hu, W.; Zheng, Z.-G.; Wu, S.-B.; Chen, P.; Li, Q.; Lu, Y.-Q. Light-activated liquid crystalline hierarchical architecture toward photonics. Adv. Opt. Mater. 2019. [CrossRef]

26. Kventsel, G.F.; Lembrikov, B.I. Two-wave mixing on the cubic non-linearity in the smectic A liquid crystals. Liq. Cryst. 1994, 16, 159-172. [CrossRef]

27. Kventsel, G.F.; Lembrikov, B.I. Stimulated light scattering in smectic A liquid crystals. Liq. Cryst. 1995, 19, 21-37. [CrossRef] 
28. Kventsel, G.F.; Lembrikov, B.I. Self-focusing and self-trapping in smectic A liquid crystals. Mol. Cryst. Liq. Cryst. 1995, 262, 629-643. [CrossRef]

29. Kventsel, G.F.; Lembrikov, B.I. The four-wave mixing and the hydrodynamic excitations in smectic A liquid crystals. Mol. Cryst. Liq. Cryst. 1995, 262, 591-627. [CrossRef]

30. Kventsel, G.F.; Lembrikov, B.I. Second sound and nonlinear optical phenomena in smectic A liquid crystals. Mol. Cryst. Liq. Cryst. 1996, 282, 145-189. [CrossRef]

31. Lembrikov, B.I. Light interaction with smectic A liquid crystals: Nonlinear effects. HAIT J. Sci. Eng. 2004, 1, 306-347.

32. Lembrikov, B.I.; Ben-Ezra, Y. Surface plasmon polariton (SPP) interactions at the interface of a metal and smectic liquid crystal. In Proceedings of the 17th International Conference on Transparent Optical Networks (ICTON 2015), Budapest, Hungary, 5-9 July 2015; We.C4.4, 1-4.

33. Lembrikov, B.I.; Ben-Ezra, Y.; Ianetz, D. Stimulated scattering of surface plasmon polaritons (SPPs) in smectic A liquid crystal. In Proceedings of the 18th International Conference on Transparent Optical Networks (ICTON-2016), Trento, Italy, 10-14 July 2016; We.B4.2, 1-4.

34. Lembrikov, B.I.; Ianetz, D.; Ben-Ezra, Y. Metal/Insulator/Metal (MIM) plasmonic waveguide containing a smectic A liquid crystal (SALC) layer. In Proceedings of the 19th International Conference on Transparent Optical Networks (ICTON 2017), Girona, Catalonia, Spain, 2-6 July 2017; Tu.A4.3, 1-4.

35. Lembrikov, B.I.; Ianetz, D.; Ben-Ezra, Y. Nonlinear optical phenomena in Silicon-Smectic A Liquid Crystal (SALC) waveguiding structures. In Proceedings of the 20th International Conference on Transparent Optical Networks (ICTON 2018), Bucharest, Romania, 1-5 July 2018; Mo.D4.1, 1-4.

36. Lembrikov, B.I.; Ianetz, D.; Ben-Ezra, Y. Nonlinear optical phenomena in smectic A liquid crystals. In Liquid Crystals-Recent Advancements in Fundamental and Device Technologies; Choudhury, P.K., Ed.; InTech: Rijeka, Croatia, 2018; pp. 131-157.

37. Shen, Y.R. The Principles of Nonlinear Optics; Wiley: Hoboken, NJ, USA, 2003.

38. Suhara, T.; Fujimura, M. Waveguide Nonlinear-Optic Devices; Springer: Berlin, Germany, 2003.

39. Haus, H.A. Waves and Fields in Optoelectronics; Prentice Hall: Upper Saddle River, NJ, USA, 1984.

40. Moloney, J.V.; Newell, A.C. Nonlinear Optics; Westview Press: Boulder, CO, USA, 2004.

41. Vagner, I.D.; Lembrikov, B.I.; Wyder, P. Electrodynamics of Magnetoactive Media; Springer: Heidelberg, Germany, 2004.

(C) 2019 by the authors. Licensee MDPI, Basel, Switzerland. This article is an open access article distributed under the terms and conditions of the Creative Commons Attribution (CC BY) license (http://creativecommons.org/licenses/by/4.0/). 